\title{
Development and clinical application of nursing-sensitive indicators for midline catheterization care using evidence-based methods
}

\author{
Wei $\mathrm{Li}^{1}$, Yandi Wen ${ }^{1}$, Yuluo Du ${ }^{1}$, Weimei Huang ${ }^{1}$, Hongxing Ye ${ }^{1}$, Jing Cao ${ }^{1}$, Weixiang Luo ${ }^{2}$, Xueyan Liu ${ }^{1}$ \\ ${ }^{1}$ Department of Critical Care Medicine, ${ }^{2}$ Department of Nursing, Shenzhen People's Hospital, 2nd Clinical Medical College of Jinan University, 1st \\ Affiliated Hospital of Southern University of Science and Technology, Shenzhen, China \\ Contributions: (I) Conception and design: W Li; (II) Administrative support: W Luo, X Liu; (III) Provision of study materials or patients: W Li, J \\ Cao; (IV) Collection and assembly of data: Y Wen, Y Du, W Huang, H Ye; (V) Data analysis and interpretation: W Li; (VI) Manuscript writing: All \\ authors; (VII) Final approval of manuscript: All authors. \\ Correspondence to: Xueyan Liu. Department of Critical Care Medicine, Shenzhen People's Hospital, 2nd Clinical Medical College of Jinan \\ University,1st Affiliated Hospital of Southern University of Science and Technology, Shenzhen, China. Email: 1736574753@qq.com; Weixiang Luo. \\ Department of Nursing, Shenzhen People's Hospital, 2nd Clinical Medical College of Jinan University,1st Affiliated Hospital of Southern University \\ of Science and Technology Shenzhen, Shenzhen, China. Email: anggle_1974@163.com.
}

\begin{abstract}
Background Midline catheters (MCs) have been widely applied in clinical settings as they can provide painless venous access, thus improving the quality of life and reducing medical costs. Nursing-sensitive indicators (NSIs) are real and effective measures of nursing quality. Using evidence-based methods, we established the NSIs of MC care, with an attempt to provide a basis for evaluating and monitoring nursing quality for MC use.

Methods: An electronic search was performed in 5 databases including China National Knowledge Infrastructure (CNKI), Chinese Scientific Journal Database (VIP), Wanfang Data, PubMed, and Web of Science to identify studies that evaluated nursing quality during MC use. Two evaluators independently selected literature, extracted data, and evaluated the risk of bias. According to the Donabedian's structureprocess-outcome model, we divided the NSIs into 3 levels.

Results: The established NSIs for MC use included 3 indicators (i.e., structure indicators, process indicators, and outcome indicators), among which there were 3 level-2 indicators and 7 level-3 indicators at the structure level, 2 level-2 indicators and 9 level-3 indicators at the process level, and 5 level-2 indicators and 17 level-3 indicators at the outcome level.
\end{abstract}

Conclusions: The established NSIs for MC use offer a set of objective criteria for evaluating nursing performance during MC use and will help to improve nursing quality control.

Keywords: Nursing quality; sensitive indicators; evidence-based medicine; midline catheter (MC)

Submitted Nov 06, 2020. Accepted for publication Jan 22, 2021.

doi: 10.21037/apm-20-2466

View this article at: http://dx.doi.org/10.21037/apm-20-2466

\section{Introduction}

Midline catheters (MCs), typically $8-25 \mathrm{~cm}$ in length, can provide venous access. MCs have been widely applied in clinical settings as they can prevent patients from suffering pain induced by repeated puncture and lower medical costs by sparing the use of $\mathrm{X}$-ray positioning $(1,2)$.
While nursing quality evaluation is an essential component of hospital management, nursing-sensitive indicators (NSIs) are real and effective measures of nursing quality (3,4). In 1998, the American Nurses Association (ANA) created the National Database for Nursing Quality Indicators (NDNQI), first proposing the concept of NSIs, 
which quantitatively evaluate the quality of a variety of functions (e.g., nursing management and clinical practice) that specifically affect patient outcomes by assessing the processes and outcomes of nursing services. It can be used to guide nursing services by providing measurable evaluation criteria for nursing performance $(5,6)$.

The nursing quality evaluation indicators are widely used across different clinical studies, and often related to other medical factors. Therefore, determining standard NSIs for MC use will be valuable for improving nursing performance. In our current study, by using evidence-based methods, we retrieved a large number of clinical studies on MC nursing, evaluated the quality of included studies, integrated and categorized the outcome indicators of NSIs in these studies, and tried to establish NSIs for MC use.

\section{Methods}

\section{Literature screening}

\section{Inclusion criteria for selecting articles}

The inclusion criteria for articles are listed below according to category.

(I) Subjects: there was no limitation on the age, gender, or ethnicity of the study participants or on the disease/condition.

(II) Interventions: MC care was involved.

(III) Research types: various types of clinical studies including randomized controlled trials, nonrandomized controlled trials, cohort studies, and case-control studies, published in English or Chinese language, were searched.

(IV) Outcome indicators: MC-related outcome indicators included nursing before, during, and after catheterization; nursing of complications; nursing before, during, and after extubation; satisfaction; and adverse reactions.

\section{Exclusion criteria for selecting articles}

Articles were excluded for any of the following reasons: (I) full-text versions of the articles were not accessible via electronic retrieval or manual retrieval; (II) publications were overlapping; (III) key information such as interventions and outcome indicators could not be extracted; (IV) articles contained abnormal data, incomparable baseline data, and/ or incomplete data; and (V) the literature was gray literature such as dissertations and papers presented at academic conferences or workshops.

\section{Literature search strategy}

We performed a literature search for eligible studies published before July 26, 2020 from 5 databases including China National Knowledge Infrastructure (CNKI), Chinese Scientific Journal Database (VIP), Wanfang Data, PubMed, and Web of Science. The subject words, combined with free words, were used in the retrieval strategy. Both English and Chinese keywords were searched, including "MC", "MVC", "medial venous catheter or midline", "midline catheters", and "nursing". References and related journals were manually searched to obtain clinical studies on MC nursing interventions. For instance, the specific search strategy in the Web of Science was as follows: TS $=(\mathrm{MC}$ OR MVC OR medial venous catheter or midline OR midline catheters) AND TS=nursing.

\section{Literature screening and data extraction}

Two researchers independently completed literature screening. First, by reading the titles and abstracts, they ruled out articles that did not meet the inclusion requirements; articles that did not clearly meet the inclusion criteria during the preliminary screening were subjected to a full-text review and stratified into an included or excluded group. Any questions, disagreements, or inconsistencies would be discussed intensively or addressed by consultation with a third expert. Data extraction (including name of the first author, publication time, literature title, outcome indicators, and risk of bias) was conducted independently by the 2 researchers. The results were cross-checked by these 2 researchers; again, any issues that arose would be negotiated or addressed by consultation with a third researcher.

\section{Assessment of publication bias}

The risk of bias was assessed by 2 independent researchers. Study quality was assessed according to the Jadad Score (7). In this system, a score of 1-3 indicates a low-quality study while a score of 4-7 indicates a high-quality one. The following criteria were used:

(I) Randomization:

(i) Appropriate (2 points), if random sequence is generated by computational algorithms or simulation methods;

(ii) Unclear (1 point), if a trial does not provide its randomization method (1 point);

(iii) Inappropriate (0 point), if an alternate assignment method is used, such as the allocation of odd and even numbers.

(II) Randomization concealment: 
(i) Appropriate (2 points), if any allocation method that clinicians and subjects cannot predict is used, including the distribution scheme is controlled by a center or pharmacy, containers with consistent serial numbers, on-site computer control, sealed opaque envelopes, etc.;

(ii) Unclear (1 point), if only a random number table or other random allocation scheme is applied;

(iii) Inappropriate ( 0 point), if any method with predictable assignments is used, including alternate allocation, case numbers, days of the week, an open-label random number table, serial-coded envelopes, etc.;

(iv) Absent (0 point), if randomization is not used.

(III) Blinding method:

(i) Appropriate (2 points), if the same placebo form or a similar method is used;

(ii) Unclear (1 point), if it is a blinded trial without any information of blinding methods;

(iii) Inappropriate (0 point), if an inappropriate blinding method is used, such as a comparison between tablets and injections.

(IV) Withdrawal and exit:

(i) The number and reasons of patients withdrawing or exiting are described (1 point);

(ii) Otherwise (0 point).

The methodological quality of observational studies (e.g., case-control studies, cohort studies, case series, and case reports) were assessed by using the use the NewcastleOttawa Scale (NOS) (8), which consists of 3 main aspects: the methods used for selecting cases and controls, the comparability between the case group and the control group, and the evaluation method of exposure. Eight evaluation items were used, and the results are indicated by stars. The quality evaluation was carried out independently and cross-checked by 2 researchers, and any disagreement was settled by negotiation or by consulting a third researcher.

\section{Construction of NSIs}

In the 1960s, Donabedian proposed the structureprocess-outcome model of quality, focusing on 3 aspects including structure quality management, process quality management, and result quality management (9). The NSIs were established and validated based on this theory. As of 2014, 18 NSIs have been selected by the NDNQI (10):

(I) structure indicators including staffing mix, nursing hours per patient day, satisfaction of registered nurses, and education level of registered nurses; (II) process indicators including physical constraints and pain evaluationintervention-re-evaluation; and (III) outcome indicators including satisfaction, adverse reactions, stratified care, and compliance.

\section{Results}

\section{Results of literature search}

Out of the initial 460 articles obtained, 174 were duplicates and hence excluded from further analysis; 12 conference/ workshop papers were excluded, and another 126 articles were excluded on the basis of title and abstract. From the articles that remained, 112 articles were excluded on the basis of their full-text, for reasons of incomplete data ( $n=95)$, unrelated interventions ( $\mathrm{n}=95)$, and lack of access to the full text $(\mathrm{n}=11)$. Ultimately, 36 studies including 17 randomized controlled trials and 19 observational studies, comprising $3060 \mathrm{MC}$ users, entered the final analysis (Figure 1).

\section{General description and quality evaluation of the included articles}

All the 17 RCTs described the baseline data and declared that these data were comparable; however, none mentioned blinding or allocation concealment, and there was no withdrawal/exit data. Only 2 studies described the specific randomization method. The quality of each article was evaluated by using the Jadad score and NOS (Table 1).

\section{Establishment of NSIs for MC care}

NSIs extracted from literature were divided into 3 levels according to the structure-process-outcome theory (Table 2). The NSIs for MC care in the enrolled articles were further analyzed, and the frequency distribution of the outcome was analyzed. Among the MC users, the rate of care for complications reached $100 \%$, and the rate of nursing before catheterization reached $50 \%$. During the proactive nursing, the complications associated with MC infusion and the estimated cost of the treatment were carefully explained to the patients and their families. Informed consent was obtained. The rate of nursing (mainly the aseptic techniques applied according to clinical standards) during catheterization reached $47.2 \%$, and the rate of nursing (including timely dressing change, catheter cleansing, 


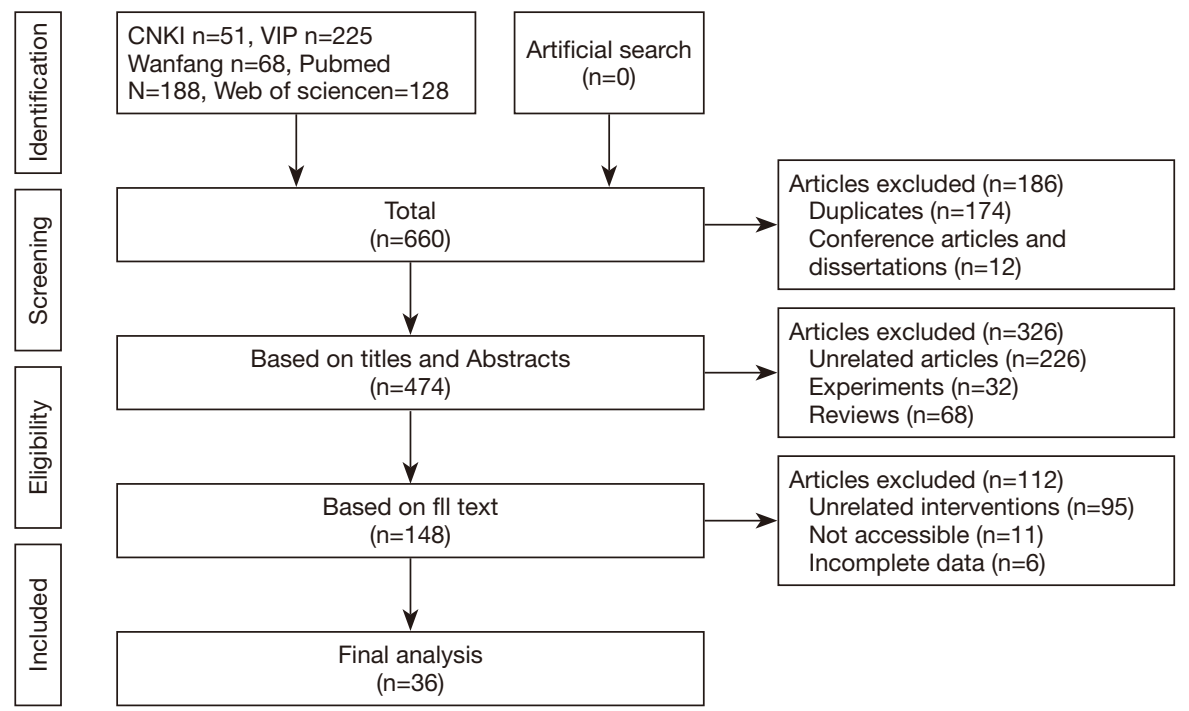

Figure 1 Literature search and screening flowchart.

Table 1 Basic information of the enrolled studies

\begin{tabular}{|c|c|c|c|c|c|c|c|}
\hline Studies included & Research type & $\mathrm{n}$ & Mode of nursing & $\begin{array}{l}\text { Duration of } \\
\text { indwelling (d) }\end{array}$ & $\begin{array}{c}\text { Site of } \\
\text { catheterization }\end{array}$ & $\begin{array}{c}\text { Method of } \\
\text { catheterization }\end{array}$ & $\begin{array}{c}\text { Quality } \\
\text { evaluation }\end{array}$ \\
\hline Li 2020, (11) & RCT & 100 & - & - & $A$ and $B$ & & 2 \\
\hline Wang 2020, (13) & RCT & 60 & - & - & - & - & 2 \\
\hline Xu 2020, (14) & RCT & 70 & - & $23.22 \pm 3.51$ & - & - & 2 \\
\hline Yang 2020, (16) & RCT & 50 & - & - & - & - & 1 \\
\hline Dong 2019, (17) & $\mathrm{RCT}$ & 93 & - & $3-47$ & - & - & 1 \\
\hline Gao 2019, (18) & $\mathrm{RCT}$ & 60 & Clinical nursing & - & - & - & 1 \\
\hline Gu 2019, (19) & Observational study & 56 & - & $10-50$ & - & 1 & a \\
\hline Sun 2019, (23) & $\mathrm{RCT}$ & 124 & - & $31.8 \pm 2.4$ & - & & 1 \\
\hline Wang 2019, (24) & $\mathrm{RCT}$ & 116 & - & - & - & 1 & 1 \\
\hline Yu 2019, (25) & Observational study & 1 & - & - & $\mathrm{C}$ & - & $b$ \\
\hline Zhang 2019, (26) & $\mathrm{RCT}$ & 110 & - & $7-55$ & $A, B$, and $C$ & & 2 \\
\hline Zhao 2019, (27) & Observational study & 1 & - & 49 & $\mathrm{C}$ & - & $b$ \\
\hline Hong 2018, (28) & $\mathrm{RCT}$ & 200 & - & $17.97 \pm 9.55$ & $\mathrm{C}$ & & 1 \\
\hline Lin 2018, (29) & Observational study & 12 & - & 22 & $\mathrm{C}$ & 1 & 2 \\
\hline Liu 2018, (30) & $\mathrm{RCT}$ & 136 & - & - & A & 1 & 1 \\
\hline
\end{tabular}

Table 1 (continued) 
Table 1 (continued)

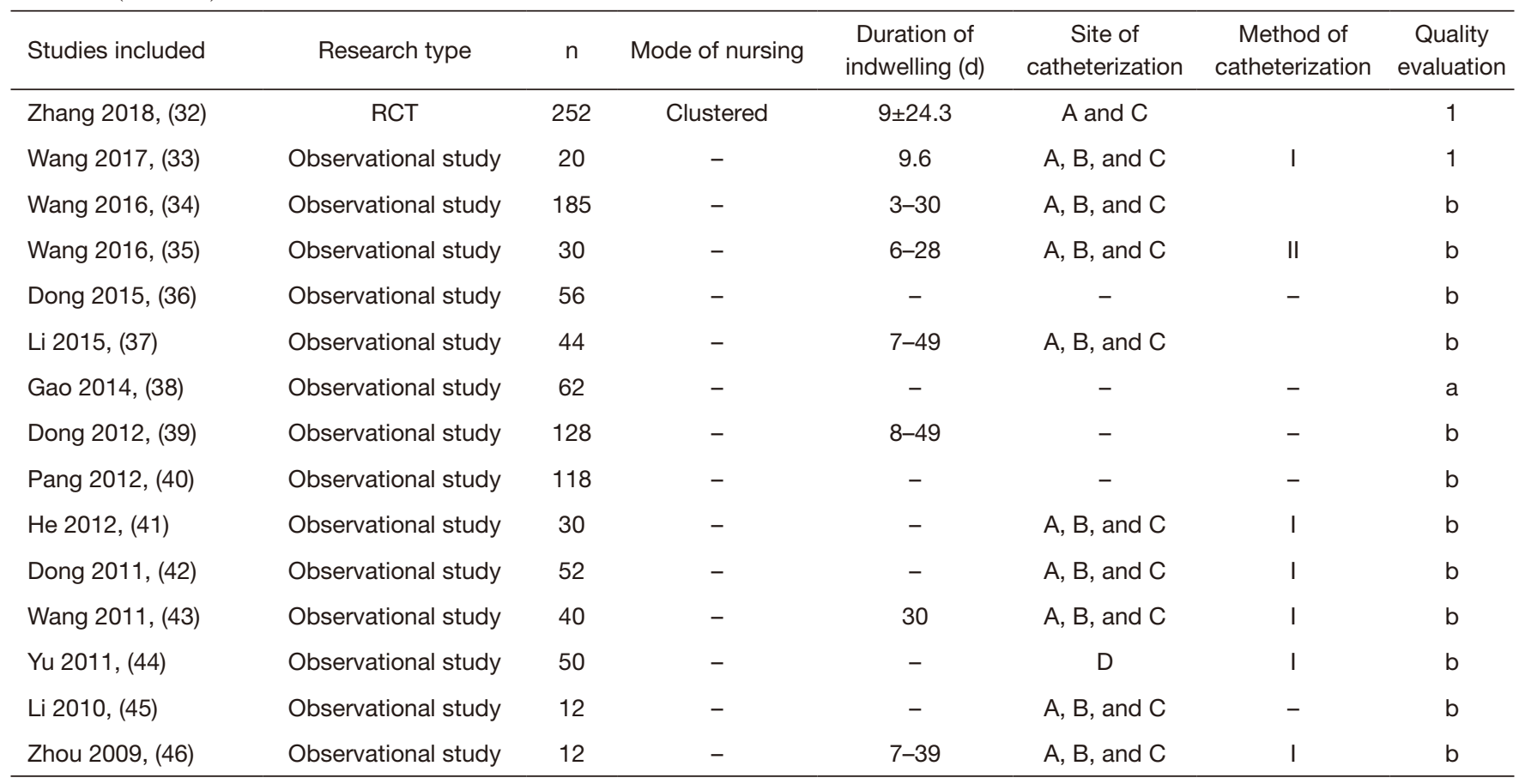

$\mathrm{A}=$ median cubital vein; $\mathrm{B}=$ cephalic vein; $\mathrm{C}=$ basilic vein; $\mathrm{D}=$ anterior cubital vein; I = Seldinger technique; II = ultrasound-guided Seldinger technique; $a=4$ stars (according to NOS); $b=3$ stars (according to NOS); $1=1$ point (according to Jadad score). RCT, randomized controlled trial.

Table 2 Nursing-sensitive indicators (NSIs) for midline catheters (MCs) care

\begin{tabular}{lcc}
\hline NSIs & $\mathrm{n}$ & Frequency \\
\hline Nursing of complications & 36 & $100.0 \%$ \\
Nursing before catheterization & 18 & $50.0 \%$ \\
Nursing during catheterization & 17 & $47.2 \%$ \\
Nursing after catheterization & 17 & $47.2 \%$ \\
Patient satisfaction & 11 & $30.6 \%$ \\
Mental health nursing & 3 & $8.3 \%$ \\
Extubation nursing & 2 & $5.6 \%$ \\
Average cost of infusion tools & 2 & $5.6 \%$ \\
Patient's compliance & 2 & $5.6 \%$ \\
Pain management nursing & 2 & $5.6 \%$ \\
Health education & 2 & $5.6 \%$ \\
Puncture success rate & 2 & $5.6 \%$ \\
Quality of life & 1 & $2.8 \%$ \\
Total number of puncture attempts & 1 & $2.8 \%$ \\
Catheter maintenance compliance rate & 1 & $2.8 \%$ \\
Wound healing time & 1 & $2.8 \%$ \\
\hline
\end{tabular}

catheter sealing, and catheter care) after catheterization was also $47.2 \%$ (Table 3).

\section{Clinical application of NSIs for MC care}

No clinical guideline or expert consensus on NSIs for $\mathrm{MC}$ care has been established. As seen in our analysis on the NSIs for MC care, the quality of NSIs for MC care varied, was highly heterogeneous across different studies, and the relevant indicators in the literature showed low standardization. Therefore, the methods of evidence-based medicine were employed in our current study to make the NSIs for MC care more standardized, uniform, and scientific, and thus better able to guide clinical practice.

\section{Discussion}

\section{Strength of our current study}

In China, research on NSIs is still in the exploratory stage, and NSIs for specific disciplines have not yet been established. Based on Donabedian's structure-processoutcome model and by employing the methods of evidence- 
Table 3 Frequencies of nursing-sensitive indicators (NSIs) for midline catheters (MCs) care

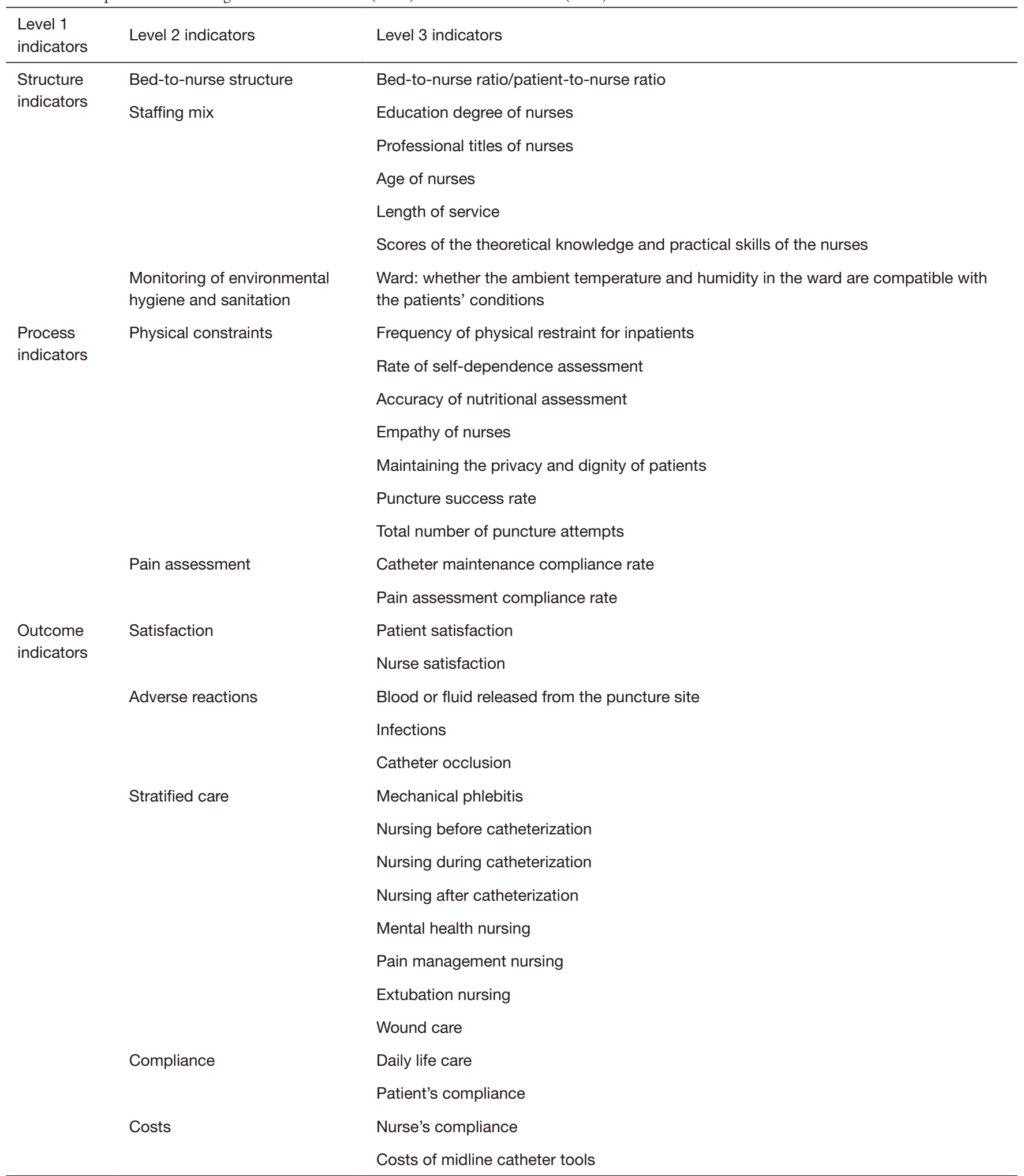


based medicine, this study is the first to analyze NSIs for MC care and establish the NSIs for MC care at the structure, process, and outcome levels, with an attempt to enrich the concept of high-quality nursing and thus improve the quality of nursing services in clinical settings.

\section{Limitations of our current study}

The analysis of the NSIs was based on literature review and data integration. Only Chinese-language articles were included in this analysis, and the overall methodological quality of these studies was unsatisfactory. Finally, the NSIs used in these articles had variable quality and were not standardized.

\section{Prospects}

To make the NSIs for MC care more suitable for clinical needs and to improve our understanding of clinical care, we will further screen and optimize the relevant indicators by (I) establishing an NSI research group, (II) adopting the 3-round Delphi method, (III) convening expert consensus meetings, and (IV) carrying our large-scale, multicenter clinical research to validate the clinical value of the final NSIs. We hope the new NSIs for MC care will be further applied in clinical settings and thus offer objective evaluation standards for nursing services and the improvement of nursing quality.

\section{Acknowledgments}

Funding: None.

\section{Footnote}

Conflicts of Interest: All authors have completed the ICMJE uniform disclosure form (available at http://dx.doi. org/10.21037/apm-20-2466). The authors have no conflicts of interest to declare.

Ethical Statement: The authors are accountable for all aspects of the work in ensuring that questions related to the accuracy or integrity of any part of the work are appropriately investigated and resolved.

Open Access Statement: This is an Open Access article distributed in accordance with the Creative Commons Attribution-NonCommercial-NoDerivs 4.0 International
License (CC BY-NC-ND 4.0), which permits the noncommercial replication and distribution of the article with the strict proviso that no changes or edits are made and the original work is properly cited (including links to both the formal publication through the relevant DOI and the license). See: https://creativecommons.org/licenses/by-nc-nd/4.0/.

\section{References}

1. Dumont C, Getz O, Miller S. Evaluation of midline vascular access: a descriptive study. Nursing 2014;44:60-6.

2. Toor H, Bowen I, Zampella B, et al. Efficacy of Trauma Catheter and Mushroom Tip Catheter in Evacuation of Chronic Subdural Hematoma and Complications of Drain Placement. Cureus 2019;11:e5123.

3. Burston S, Chaboyer W, Gillespie B. Nurse-sensitive indicators suitable to reflect nursing care quality: a review and discussion of issues. J Clin Nurs 2014;23:1785-95.

4. Liu SW, Singer SJ, Sun BC, et al. A conceptual model for assessing quality of care for patients boarding in the emergency department; structure-process-outcome. Acad Emerg Med 2011;18:430-5.

5. Isis $M$. The national database of nursing quality indicators (NDNQI). Online J Issues Nurs 2007;12:7.

6. American Nurses Association. NDNQI: transforming data into quality Care. Available online: www.nurisngquality. org, 2010.

7. Jadad AR, Moore RA, Carroll D, et al. Assessing the quality ofreports of randomized clinical trial:is blinding necessary. Control Clin Trials 1996;17:1-12.

8. Stang A. Critical evaluation of the Newcastle-Ottawa scale for the assessment of the quality of nonrandomized studies in meta analyses. Eur J Epidemiol 2010;25:603-5.

9. Donabedian A. Quality of care: problems of measurement. II. Some issues in evaluating the quality of nursing care. Am J Public Health Nations Health 1969;59:1833-6.

10. Chee J. Representative tasks that define the essence of the nursing domain: using nursing sensitive indicators for deliberate practice. Nurse Education in Practice 2014;14:327-8.

11. Li SW, Jing YN, Yang XF, et al. Application of midline venous catheterization in critically ill patients undergoing neurosurgery. China Health Care and Nutrition 2020;30:102.

12. Liu YF, Li CH, Pan YQ. Application of comprehensive nursing interventions in patients with gastric cancer undergoing midline catheterization. Gansu Science and Technology 2020;36:107-9. 
13. Wang XZ. Application of midline catheter in transfusion of premature and very-low-birth-weight infants: an analysis of 30 cases. Medical Diet and Health 2020;18:151-2.

14. $\mathrm{Xu} \mathrm{D}, \mathrm{Wu}$ MR, Cao LJ. Application and nursing of midline catheter intravenous infusion in critically ill patients in the digestive departments. Electronic Journal of Practical Clinical Nursing Science 2020;5:30-1.

15. Yan P, Wang L. Application effect of midline catheter in patients with cerebral hemorrhage. Clinical Research and Practice 2020;5:155-7.

16. Yang LJ. Application of PDCA cycle in the maintenance of midline catheter. Journal of Clinic Nursing's Practicality 2020;5:21-3.

17. Dong LL. Effectiveness of high-quality nursing for patients with neurological diseases treated by intravenous infusion with midline catheter (J). Contemporary Medical Symposium 2019;17:279-80.

18. Gao YC, Yang Y. The Application of Clinical Nursing Pathway in Midline Catheter Nursing. Journal of Clinic Nursing's Practicality 2019;4:1-3.

19. Gu XL. Complications associated with midline catheterization and their nursing. Journal of Clinic Nursing's Practicality 2019;4:81.

20. He XL, Huang QJ, Li GH. Application of ultrasoundguided Seldinger technique in midline catheterization in HIV/AIDS patients. Chinese Community Doctors 2019;35:131, 134.

21. Jing YN, Li SW, Sun QS, et al. Causes of complications associated with midline catheterization in neurosurgery wards and their nursing. China Health Care and Nutrition 2019;29:245.

22. Li JZ, Dai YF, Feng W. Application of midline catheter in intravenous infusion. Diet Health 2019;6:177-8.

23. Sun QY. Effectiveness of comprehensive nursing for patients with neurological diseases treated by intravenous infusion with midline catheter. Contemporary Medical Symposium 2019;17:243-80.

24. Wang PP, Tong YJ, Sun YL. Complications of medline catheterization for intravenous infusion and their nursing. China Health Vision 2019;(17):167, 169.

25. Yu T, Liu WW. Nursing during recanalization of occluded modified midline catheter: report of one case. Nursing of Integrated Traditional Chinese and Western Medicine 2019;5:184-6.

26. Zhang J, He JY. Satisfaction about the modified midline catheter for intravenous infusion. Journal of Clinic Nursing's Practicality 2019;4:139, 141.

27. Zhao N, Guan LL, Zhang XM, et al. Clinical nursing of midline catheterization in patients with pulmonary infection after renal transplantation. Practical Journal of Organ Transplantation 2019;7:288-9.

28. Hong XH, Zhang HR, Zhou Q, et al. Application of indwelling needle and modified medium-length catheter in critical stroke patients. Contemporary Medicine 2018;24:5-8.

29. Lin JH, Zhu J, Zhao LN. Nursing of midline catheter for intravenous infusion in general surgery. Journal of Clinical Medical Literature (Electronic Edition) 2018;5:108.

30. Liu JJ, Zhang J, Tan L. Comparison on the application of modified midline catheters and PICC applied in patients with gastrointestinal tumors in perioperative period. Nursing Practice and Research 2018;15:143-5.

31. Yao Q. Influence of medical care integration on compliance and therapeutic effect of patients with indwelling catheter. Chinese and Foreign Medical Research 2018;16:177-9.

32. Zhang DX, Li XL, Ma YJ. Clustered intervention strategy in reducing the complications of midline catheterization in cancer patients. Journal of Clinical Medical Literature (Electronic Edition) 2018;5:118-9.

33. Wang SM, Wu KL, Zhang M, et al. Application and nursing of medium-length catheters in patients with tuberculous Meningitis. Medical Information, 2017;30:112-3.

34. Wang Q, FU Rong, Sun J, et al. Application of modified peripheral vein midline catheters in patients with neurological diseases. Journal of Nursing 2016;23:72-5.

35. Wang X. Nursing of ultrasound-guided Sedinger technique in departments of neurology. Today Nurse 2016;(6):39-40.

36. Dong H, Deng J, Huamg QQ, et al. Causes and nursing of unplanned extubation of indwelling midline venous catheter in neurosurgery. Psychological Doctor 2015;21:145-6.

37. Li AX, Feng YL, Feng LP. Application and nursing of midline catheter in infusion of patients after thoracic surgery: report of 44 cases. Medical Information 2015;(34):151.

38. Gao LH. Nursing of midline catheterization for intravenous infusion in departments of pediatric neurology. Chinese Journal of Practical Nursing 2014;30:86.

39. Dong H, Wang J, Li L, et al. Causes and nursing of unplanned extubation of midline venous catheters in patients with craniocerebral injury. World Health Digest 2012;9:262-3.

40. Pang Y, Yang NL, Dai XD. Complications of medline catheterization for intravenous infusion and their nursing. 
Journal of North Pharmacy 2012;9:127-40.

41. He JF, Li JH, Xue H. Application of midline catheter in perioperative patients. Frontiers of Medicine 2012;2:39-40.

42. Dong YH. Application of midline catheter in perioperative nutritional support. Health Must-read Magazine 2011;(7):226-7.

43. Wang YP. Nursing of midline venous catheter for infusion therapy. Chinese Nursing Research 2011;25:1932-3.

44. Yu L, Tian JP. Application of midline catheter in severe

Cite this article as: Li W, Wen Y, Du Y, Huang W, Ye H, Cao J, Luo W, Liu X. Development and clinical application of nursingsensitive indicators for midline catheterization care using evidence-based methods. Ann Palliat Med 2021;10(1):425-433. doi: 10.21037/apm-20-2466 patients in departments of neurology. Journal of Clinical Medicine in Practice 2011;15:41-2.

45. Li Y, Xia JW. Application of midline catheter for intravenous infusion in digestive departments. Nursing and Rehabilitation Journal 2010;9:877-9.

46. Zhou XH, Zhou Y. Application of midline catheter in clinical intravenous infusion. Nursing Journal of Chinese People's Liberation Army 2009;26:58-9.

(English Language Editor: J. Gray) 